\title{
Exact Solution to the Linearized Poisson-Boltzmann Equation for Spheroidal Surfaces
}

The Poisson- Boltzmann equation governing the electrostatic potential distribution around charged spheroidal surfaces immersed in an electrolyte solution is derived. We show that if the surface potential is low, the linearized Poisson- Boltzmann equation can be solved exactly. Both constant surface potential and constant amount of surface charges are considered, the latter includes conductive and nonconductive surfaces. The results of numerical simulation reveal that for a spheroidal surface of constant potential the distance between the equal-potential surface to the charged surface is inversely proportional to the curvature of the latter. In other words, the thickness of the double layer is position dependent. For a nonconductive surface, the surface potential is inversely proportional to the curvature, and for a conductive surface, the charge density is proportional to the curvature of the surface. The present analysis is appropriate for a relatively thick double layer and/or a small focus. @ 1996 Academic Press, Inc.

The distribution of electrostatic potential around a charged surface immersed in an electrolyte solution is governed by the Poisson-Boltzmann equation (PBE). For a spheroidal surface, the solution to PBE involves the spheroidal wave functions. Although these functions have been investigated extensively $(1,2)$, their applications in colloidal science are still very limited. Fair and Anderson (3) and Feng and Wu (4) examined the electrophoretic behavior of an ellipsoidal particle. The analyses were based on the assumption that an electrical double layer is thin, and the electrostatic potential distribution can be approximated by that of a planar surface. The spheroidal wave functions were used by Yoon and Kim (5) to analyze the electrophoretic behavior of spheroidal particles. Their solution is adequate for the case in which the thickness of a double layer is comparable to the particle size. The eigenvalues and the coefficients in the expression for the electrical potential distribution needed to be evaluated through finding the roots of a transcendental equation. The numerical procedure is time-consuming and needs to be conducted for each value of $c$, the product of the Debye length and the focus of a particle.

In the present study, an attempt is made to recover the analytical solution of the PBE at a low electrical potential for a charged spheroidal surface. A spheroidal wave function expansion different from that adopted by Yoon and Kim (5) is chosen to improve its computing efficiency. The effect of surface conditions on the electrical potential distribution is discussed. Both constant surface potential and constant amount of surface charges are considered, the latter includes conductive and nonconductive surfaces. The Helmholtz free energy of the system under consideration is also derived.

The electrostatic potential distribution for a charged surface immersed in an electrolyte solution is described by the Poisson-Boltzmann equation. If the potential is low, it can be approximated by

$$
\nabla^{2} y=\kappa^{2} y,
$$

where $y=e \Psi / k_{\mathrm{B}} T$, and $\kappa^{2}=8 \pi e^{2} I / \epsilon k_{\mathrm{B}} T$. In these expressions, $\nabla^{2}$ is the
Laplace operator, $\Psi$ denotes the electrostatic potential, $\epsilon$ is the dielectric constant, $\kappa$ and $k_{\mathrm{B}}$ are the reciprocal Debye length and the Boltzmann constant, respectively, $T$ is the absolute temperature, and $e$ and $I$ are, respectively, the elementary charge and the ionic strength. In spheroidal coordinates $(6),[1]$ can be written as

$$
\frac{\partial}{\partial \eta}\left[\left(1-\eta^{2}\right) \frac{\partial y}{\partial \eta}\right]+\frac{\partial}{\partial \xi}\left[\left(\xi^{2}-j\right) \frac{\partial y}{\partial \xi}\right]-c^{2}\left(\xi^{2}-j \eta^{2}\right) y=0,
$$

where $j=1$ for a polate spheroid, $j=-1$ for an oblate spheroid, and $c=$ $\kappa a$. Here, $\xi$ and $\eta$ are the radial and the angle coordinates, respectively, $(1+j) / 2 \leqslant \xi<\infty$ and $-1 \leqslant \eta \leqslant 1$, and $a$ is the distance between the focus and the center of a particle. The oblate coordinates can be transformed into the prolate coordinates by letting $\xi= \pm i \xi, c=\mp i c$, and $i=\sqrt{-1}$. Assuming that $y=R(\xi) S(\eta)$, [2] leads to

$$
\begin{gathered}
\frac{\partial}{\partial \eta}\left[\left(1-\eta^{2}\right) \frac{\partial S_{n}}{\partial \eta}\right]+\left(\lambda_{n}+j c^{2} \eta^{2}\right) S_{n}=0 \\
\frac{\partial}{\partial \xi}\left[\left(1-j \xi^{2}\right) \frac{\partial R_{n}}{\partial \xi}\right]+j\left(\lambda_{n}+c^{2} \xi^{2}\right) R_{n}=0,
\end{gathered}
$$

where $\lambda_{n}, n=0,1, \ldots$, are the eigenvalues, and $S_{n}$ and $R_{n}$ are the associated eigenfunctions.

If $c^{2}$ is small, we can expand $S_{n}$ and $\lambda_{n}$ as

$$
\begin{gathered}
S_{n}=\sum_{r=0}^{\infty} S_{n, r}\left(j c^{2}\right)^{r} \\
\lambda_{n}=\sum_{r=0}^{\infty} \lambda_{n, r}\left(j c^{2}\right)^{r} .
\end{gathered}
$$

Since as $c \rightarrow 0$ [3a] reduces to the Legendre equation, both [4] and [5] imply that $S_{n, 0}=P_{n}$ and $\lambda_{n, 0}=n(n+1)$. For spheroidal surfaces, $-1 \leqslant$ $\eta \leqslant 1$. Substituting [4] and [5] into [3a] yields the recursive equation

$$
\frac{\partial}{\partial \eta}\left[\left(1-\eta^{2}\right) \frac{\partial S_{n, r}}{\partial \eta}\right]+\eta^{2} S_{n, r-1}+\sum_{p=0}^{r} \lambda_{n, p} S_{n, r-p}=0, \quad r \geqslant 1
$$

where $P_{n}$ is the Legendre polynomial of first kind of order $n$. Suppose that $S_{n, r}$ can be expanded as

$$
S_{n, r}=\sum_{s=0,1}^{\infty} a_{n, r, s} P_{s},
$$

where $s$ is even if $n$ is even and odd if $n$ is odd. The Legendre polynomial satisfies the relation 


$$
\eta P_{s}(\eta)=\frac{s+1}{2 s+1} P_{s+1}(\eta)+\frac{s}{2 s+1} P_{s-1}(\eta)
$$

Substituting [7] and [8] into [6] gives

$$
\begin{gathered}
\lambda_{n, r}=-\left[\alpha(n) a_{n, r-1, n+2}+\beta(n) a_{n, r-1, n}+\gamma(n) a_{n, r-1, n-2}\right] \\
a_{n, r, s}=\frac{1}{s(s+1)-\lambda_{n, 0}} \\
\left\{\alpha(s) a_{n, r-1, s+2}+\beta(s) a_{n, r-1, s}+\gamma(s) a_{n, r-1, s-2}+\sum_{p=1}^{r} \lambda_{n, p} a_{n, r-p, s}\right\}, \\
a_{n, r, s}=0 \quad s=n .
\end{gathered}
$$$$
s \neq n \quad[9 \mathrm{~b}]
$$

In these expressions, $\alpha(s)=(s+2)(s+1) /(2 s+5) /(2 s+3), \beta(s)=$ $\left(2 s^{2}+2 s-1\right) /(2 s-1) /(2 s+3)$, and $\gamma(s)=s(s-1) /(2 s-3) /(2 s$ $-1)$. Note that both $\lambda_{n, r}$ and $S_{n, r}$ have a finite number of terms.

According to $(1,7)$, the radial functions can be defined as

$$
R_{n}(\xi)=\frac{\pi}{2} \int_{1}^{\infty} \exp (-c \xi \eta) S_{n}(\eta) d \eta
$$

Based on the property of the Legendre function, we have

$$
\int_{1}^{\infty} \exp (-x y) P_{n}(x) d x=\frac{2}{\pi} K_{n}(y)
$$

where $K_{n}$ is the modified spherical Bessel function of the third kind of order $n(8)$, which can be expressed as

$$
K_{n}(x)=\sqrt{\frac{\pi}{2 x}} k_{n+1 / 2}(x)
$$

In this expression, $k_{n}(x)$ is the modified Bessel function of the second kind. Substituting [11] into [10], the radial function becomes

$$
R_{n}(c, \xi)=\sum_{r=0}^{\infty} \sum_{s=0,1}^{\infty} a_{n, r, s}\left(j c^{2}\right)^{r} K_{n}(c \xi)
$$

If the argument is small, [12] is inappropriate. In this case, Aoi (7) suggests using

$$
R_{2 m}(c, \xi)=\sum_{p=0}^{\infty} \nu_{2 p} K_{p}\left[\frac{c}{2}\left(\xi+\sqrt{\xi^{2}-j}\right)\right] I_{p}\left[\frac{j c}{2}\left(\xi-\sqrt{\xi^{2}-j}\right)\right]
$$

where $I_{p}$ is the modified spherical Bessel function of the first kind of order $p$, and

$$
\begin{aligned}
\nu_{2 p}=(2 p & +1) \sum_{s=0}^{p} \sum_{i=s}^{p} \sum_{r=0}^{\infty} \\
& \times \frac{(-1)^{i+(1-j) p / 2} 2^{2 s}(i+s) !(2 i) !(p+i) !}{(i-s) !(2 i+2 s+1) !(i !)^{2}(p-i) !} a_{2 m, r, 2 s}\left(j c^{2}\right)^{r} .
\end{aligned}
$$

The boundary condition at the solid surface depends on its nature. Two types of surface are examined, with constant surface potential and constant amount of surface charges.
For a surface of constant potential the boundary condition associated with [2] is

$$
y=y_{s} \quad \text { at } \xi=\xi_{s} .
$$

Solving [2] subject to this expression yields

$$
y=\sum_{m=0}^{\infty} y_{s} A_{2 m} \frac{R_{2 m}(c, \xi)}{R_{2 m}\left(c, \xi_{s}\right)} S_{2 m}(c, \eta)
$$

where

$$
A_{2 m}=\sum_{r=0}^{\infty} a_{2 m, r, 0} c^{2 r} / \sum_{w=0}^{\infty}\left[\left(\sum_{r=0}^{\infty} a_{2 m, r, 2 w} c^{2 r}\right)^{2} /(4 w+1)\right] .
$$

Suppose that a certain amount of fixed charges are distributed uniformly over a nonconductive surface. In this case, the boundary condition at the surface is

$$
\vec{n} \cdot \vec{\nabla} y=-\frac{4 \pi e \sigma}{\epsilon k_{\mathrm{B}} T},
$$

where $\vec{n}$ denotes the unit outer-normal vector of the surface and $\vec{\nabla}$ and $\sigma$ are, respectively, the gradient operator and the surface charge density. The solution of [2] subject to [16] is

$$
y=\sum_{m=0}^{\infty} \sigma^{\prime} B_{2 m} \frac{R_{2 m}(c, \xi)}{R_{2 m}^{\prime}\left(c, \xi_{s}\right)} S_{2 m}(c, \eta)
$$

where

$$
\begin{gathered}
B_{2 m}=-\int_{0}^{1} h_{s}^{\prime} S_{2 m}(c, \eta) d \eta / \sum_{w=0}^{\infty}\left(\left(\sum_{r=0}^{\infty} a_{2 m, r, 2 w} c^{2 r}\right)^{2} /(4 w+1)\right. \\
\sigma^{\prime}=\frac{4 \pi e \sigma a}{\epsilon k_{\mathrm{B}} T} \\
h_{s}^{\prime}=\sqrt{\frac{\xi_{s}^{2}-j \eta^{2}}{\xi_{s}^{2}-j}}
\end{gathered}
$$

For a conductive surface, since charges are allowed to migrate, an equilibrium distribution will be achieved. The charges are distributed such that the potential is the same at each point on the surface. The boundary condition at the surface can be written as

$$
\vec{n} \cdot \vec{\nabla} y=-\frac{4 \pi e \sigma(\eta)}{\epsilon k_{\mathrm{B}} T}
$$

The solution of [2] has the same form as [15], except that $y_{s}$ needs to be determined from the total amount of surface charges $q$. Integrating [18] gives

$$
\int_{0}^{1}\left(\frac{\partial y}{\partial \xi}\right)_{\xi=\xi_{s}} d \eta=-\frac{e q}{a\left(\xi_{s}^{2}-j\right) \epsilon k_{\mathrm{B}} T} \equiv-q^{\prime}
$$

Substituting [15] into this expression, we obtain 

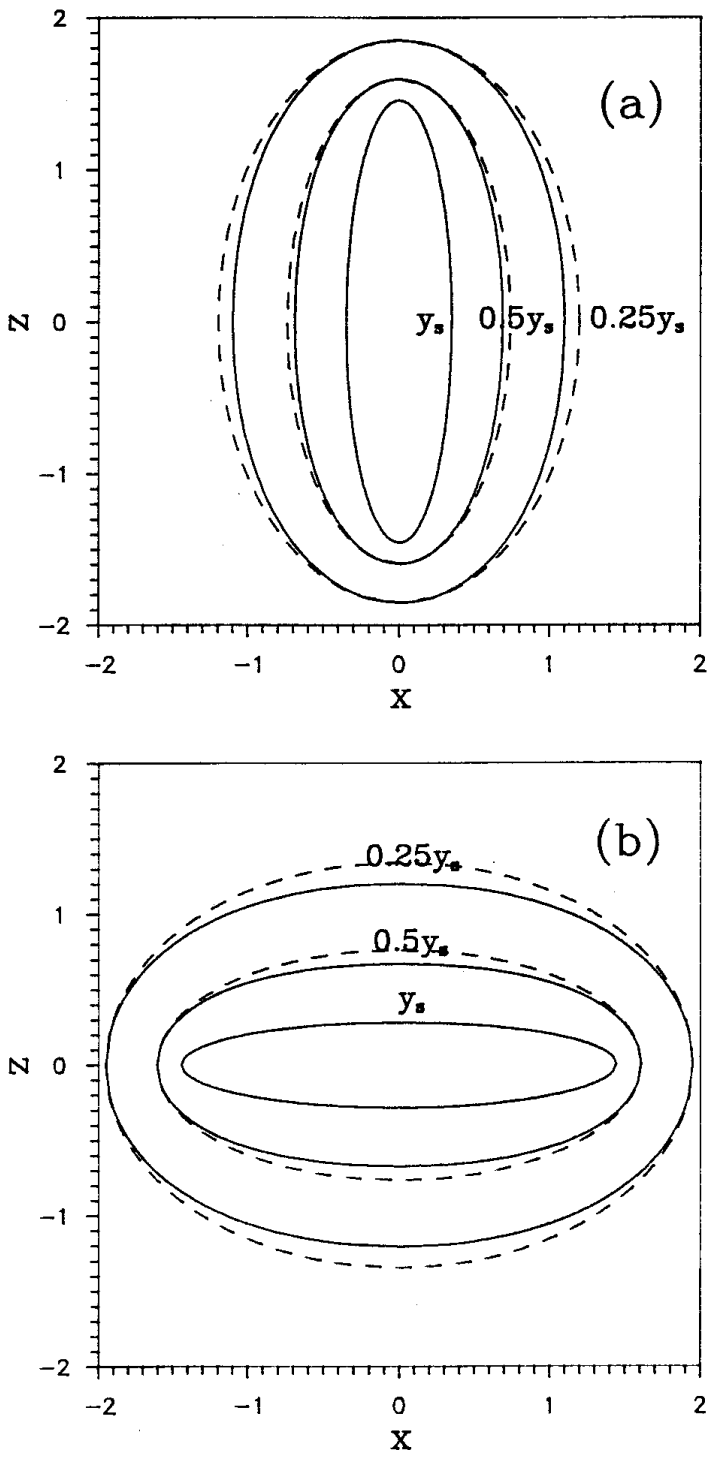

FIG. 1. Projection of equal-potential surfaces on the $z-x$ plane for a prolate spheroidal surface with $\xi_{s}=1.03$ and $c^{2}=2(\mathrm{a})$, and for an oblate spheroidal surface with $\xi_{s}=0.2$ and $c^{2}=2(\mathrm{~b})$. The dashed lines are the equal- $\xi_{s}$ planes.

$$
y_{s}=-\frac{q^{\prime}}{\sum_{m=0}^{\infty} A_{2 m}\left(\left(\sum_{r=0}^{\infty} a_{2 m, r, 0} c^{2 r}\right) R_{2 m}^{\prime}\left(c, \xi_{s}\right) / R_{2 m}\left(c, \xi_{s}\right)\right) .}
$$

Substituting [15] into [18] yields the charge distribution on the surface as

$$
\sigma^{\prime}=-\sum_{m=0}^{\infty} y_{s} A_{2 m} \frac{R_{2 m}^{\prime}\left(c, \xi_{s}\right) S_{2 m}(c, \eta)}{R_{2 m}\left(c, \xi_{s}\right) h_{s}^{\prime}}
$$

The definition of the perturbation parameter $c$ reveals that it is the ratio (focus/Debye length). Since the expansions of $S_{n}$ and $R_{n}$ in [4] and [10] require that $c$ is small, the present analysis is appropriate for a relatively thick double layer and/or a small focus. The latter implies that a particle is close to a sphere.

Figure 1 shows the variation of the equal-potential surface for a spheroidal surface of constant potential. These figures reveal that the distance between the equal-potential surface to the charged surface is inversely proportional to the curvature of the latter. In other words, the thickness of the double layer is position dependent. However, the greater the $\xi_{s}$, the smaller the difference in the distance. This is expected, since as $\xi_{s}$ increases, a spheroidal surface approaches a spherical surface. Note that the equal-potential surface is not equal to the $\xi$ coordinate surface, the variation of potential in the $\eta$ direction cannot be omitted.

The variation in the potential over a nonconductive spheroidal surface is presented in Fig. 2a. As can be seen from this figure, the surface potential is inversely proportional to the curvature. Figure $2 b$ shows the variation in the surface charge density as a function of the position on a conductive spheroidal surface. The charge density is proportional to the curvature of the surface, as expected.
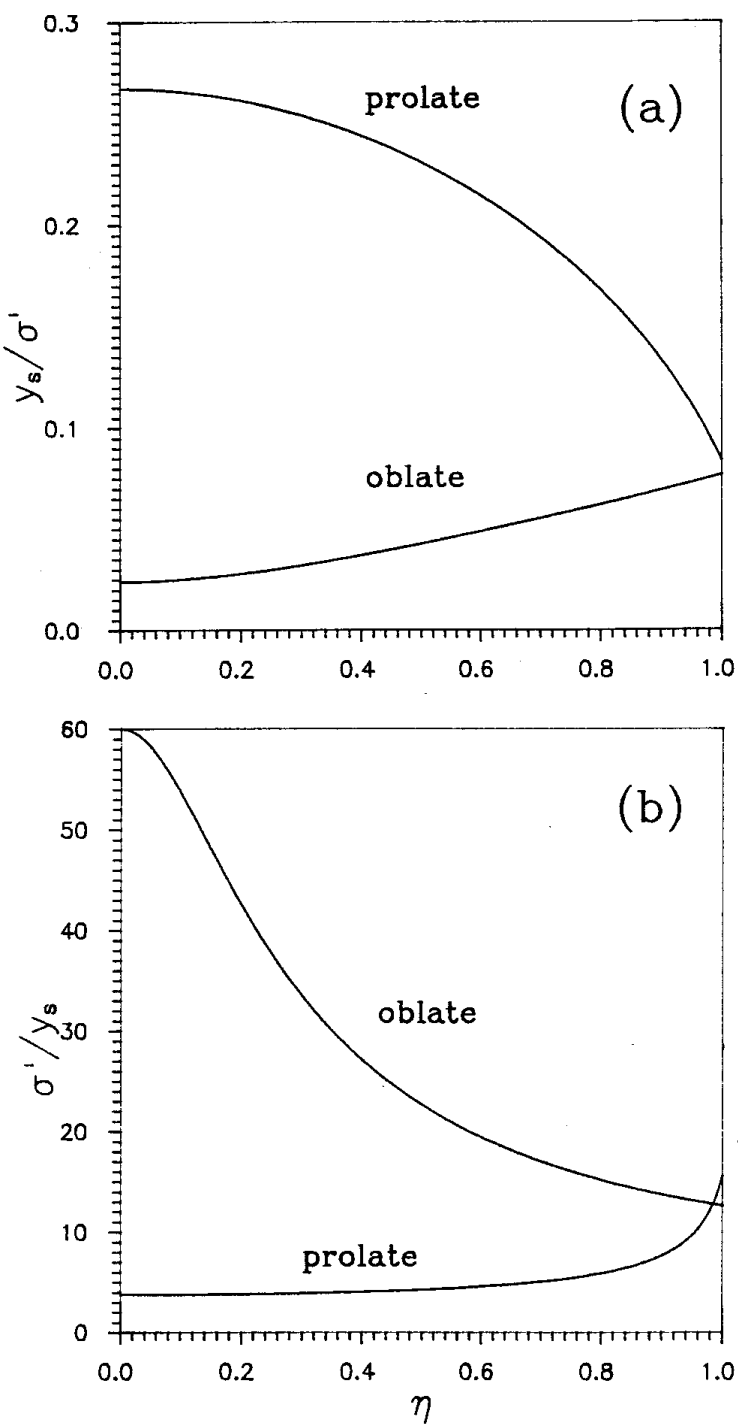

FIG. 2. (a) Variation in the surface potential as a function of $\eta$ for a nonconductive spheroidal surface. Parameters used are $\xi_{s}=1.03$ and $c^{2}=$ 2 for a prolate spheroid, and $\xi_{s}=0.2$ and $c^{2}=2$ for an oblate spheroid. (b) Variation in the distribution of surface charges as a function of $\eta$ for a conductive spheroidal surface. Parameters used are $\xi_{s}=1.03$ and $c^{2}=$ 2 for a prolate spheroid, and $\xi_{s}=0.2$ and $c^{2}=2$ for an oblate spheroid. 
It is interesting to note that the distribution of charge density in Fig. 2b for an oblate spheroidal surface has an inflection point, which does not exist for a prolate spheroid surface. This is also shown in the work of Yoon and $\operatorname{Kim}(5)$.

If the state of an uncharged surface is chosen as the reference state, the dimensionless Helmholtz free energy of the double layer $F_{e 1}$ can be calculated by

$$
F_{e 1}=\int_{0}^{\sigma^{\prime}} y_{s}\left(\sigma^{\prime}\right) d \sigma^{\prime}
$$

where $F_{e 1}=4 \pi e^{2} a f_{e 1} / \epsilon k_{\mathrm{B}}^{2} T^{2}$ and $f_{e 1}$ is the Helmholtz free energy per unit area. It can be shown that the Helmholtz free energies for constant potential, $F_{e 1, p}$, constant surface charges for a nonconductive surface, $F_{e 1, c n}$, and for a conductive surface, $F_{e 1, c c}$, are

$$
\begin{gathered}
F_{e 1, p}=-\sum_{m=0}^{\infty} \frac{y_{s}^{2}}{2} A_{2 m} \frac{R_{2 m}^{\prime}\left(c, \xi_{s}\right)}{R_{2 m}\left(c, \xi_{s}\right)} S_{2 m}(c, \eta) \\
F_{e 1, c n}=\sum_{m=0}^{\infty} \frac{\left(\sigma^{\prime}\right)^{2}}{2} B_{2 m} \frac{R_{2 m}\left(c, \xi_{s}\right)}{R_{2 m}^{\prime}\left(c, \xi_{s}\right)} S_{2 m}(c, \eta)
\end{gathered}
$$

$$
\begin{aligned}
F_{e 1, c c}=-\left(\sum_{m=0}^{\infty} \frac{\left(q^{\prime}\right)^{2}}{2} A_{2 m} \frac{R_{2 m}^{\prime}\left(c, \xi_{s}\right) S_{2 m}(c, \eta)}{R_{2 m}\left(c, \xi_{s}\right) h_{s}^{\prime}}\right) / \\
\left(\sum_{m=0}^{\infty}\left[\sum_{r=0}^{\infty} a_{2 m, r, 0} c^{2 r}\right] A_{2 m} \frac{R_{2 m}^{\prime}\left(c, \xi_{s}\right)}{R_{2 m}\left(c, \xi_{s}\right)}\right)^{2} .
\end{aligned}
$$

Note that both $F_{e 1, p}$ and $F_{e 1, c c}$ have a maximum at $\eta=1$ for prolate and at $\eta=0$ for oblate, and $F_{e 1, c n}$ has a maximum at $\eta=0$ for prolate and at $\eta=1$ for oblate.

\section{ACKNOWLEDGMENT}

This work is supported by the National Science Council of the Republic of China.

\section{REFERENCES}

1. Flammer, C., "Spheroidal Wave Functions.' Stanford Univ. Press, Stanford, CA, 1957.

2. Stratton, J. A., Morse, P. M., Chu, L. J., Little, J. D. C., and Corbato, F. J., 'Spheroidal Wave Functions.' Wiley, New York, 1956.

3. Fair, M. C., and Anderson, J. L., J. Colloid Interface Sci. 127, 388 (1989).

4. Feng, J. J., and Wu, W. Y., J. Fluid Mech. 264, 41 (1994).

5. Yoon, B. J., and Kim, S., J. Colloid Interface Sci. 128, 275 (1989).

6. Moon, P., and Spencer, D. E., "Field Theory Handbook.' SpringerVerlag, Berlin, 1961.

7. Aoi, T., J. Phys. Soc. Jpn. 10, 130 (1955).

8. Abramowitz, M., and Stegun, I. A., "'Handbook of Mathematical Functions.' Dover, New York, 1965.

JYH-PING HsU ${ }^{1}$ Bo-TAU LIU

Department of Chemical Engineering

National Taiwan University

Taipei, Taiwan 10617

Republic of China

Received March 24, 1995

\footnotetext{
${ }^{1}$ To whom correspondence should be addressed.
} 